\title{
基于作物生长模型的玉米生殖期冷害致灾因子研究
}

\author{
马玉平 ${ }^{1,2, *}$ 王石立 ${ }^{1}$ 李维京 $^{3}$ \\ ${ }^{1}$ 中国气象科学研究院, 北京 $100081 ;{ }^{2}$ 南京信息工程大学, 江苏南京 $210044 ;{ }^{3}$ 国家气候中心, 北京 100081
}

摘 要: 利用东北玉米生长模型(NEC_MaGM)结合历史冷害数据分析了东北玉米灌浆期低温和初霜冻的发生规律, 探讨了两者对玉米生长发育过程的影响与实际冷害年份之间的对应关系。结果表明，东北地区玉米灌浆期间较易出 现 $15 \sim 16^{\circ} \mathrm{C}$ 以下的急剧降温天气, 对籽粒灌浆影响很大; 出现在发育指数(DVS)为 1.9 之前且日平均温度连续 $5 \mathrm{~d}$ 低于 $16^{\circ} \mathrm{C}$ 的低温将对玉米咜存器官干重(WSO)积累造成重大影响, 其出现年份与实际冷害发生年份符合度较高, 是发生 大面积冷害的致灾因子之一; 随着气候变暖, 东北各地初霜冻日和新玉米品种的成熟期均在推迟, 但初霜冻日的年 际波动不断增大。模拟显示, 初霜冻发生在玉米成熟前的频率也在增加, 初霜冻对玉米的影响仍不容忽视; 初霜冻对 WSO 积累影响严重的年份与历史上出现冷害的一些典型年份比较一致，也是重要的冷害致灾因子之一。灌浆期低温 和初霜冻出现时间以及由此导致 WSO 的损失程度可以作为基于作物生长模型进行玉米冷害监测预测的重要指标。 同时, 作物生长模型可以成为研究农业气象灾害致灾因子的有力工具。

关键词: 玉米冷害; 灌浆期低温; 初霜冻; 作物生长模型

\section{Chilling Disaster Factors in Maize Reproductive Stage Based on Crop Growth Model}

\author{
MA Yu-Ping ${ }^{1,2, *}$, WANG Shi-Li ${ }^{1}$, and LI Wei-Jing ${ }^{3}$ \\ ${ }^{1}$ Chinese Academy of Meteorological Sciences, Beijing 100081, China; ${ }^{2}$ Nanjing University of Information Science \& Technology, Nanjing 210044 , \\ China; ${ }^{3}$ National Climate Center, Beijing 100081, China
}

\begin{abstract}
In recent years, temperature in the northeast of China has been significantly increasing with global warming. However, since planting areas of relatively late-maturing varieties of maize are shifting towards north, the frequency and intensity of maize chilling disaster are increased in some areas and some years. The prevention and mitigation of chilling disaster of maize in the Northeast depend on our understanding about chilling disaster factors and the degrees of impacts. In this paper, using North East China Maize Growth Model (NEC_MaGM) and historical chilling disaster data, we analyzed temporal and spatial variations of low temperature during the grain filling period and the first frost day, and discussed the match relations between chilling years based on the two disaster factors and actual chilling years. The results indicated that NEC_MaGM had a satisfied outcome in simulating maize growth and development. The absolute deviation at simulated tasseling and maturity stages calculated by NEC_MaGM was $3.6 \mathrm{~d}$ and $5.9 \mathrm{~d}$, respectively. The root mean square error of simulated weight of storage organ (WSO) was $839.5 \mathrm{~kg} \mathrm{ha}^{-1}$. The average temperature in grain filling stage of maize in the Northeast was $16.7-23.5^{\circ} \mathrm{C}$. However, the rapid decrease of temperature $\left(<16^{\circ} \mathrm{C}\right)$ that usually occurred in the late period of maize development stage had a great impact on grain filling. The average temperature on the first frost day in the northeast was $7.4-9.2^{\circ} \mathrm{C}$, which was below the threshold for maize growth. In addition, the first frost day in the Northeast had a trend of delay with time. However, maturity of new maize variety also delayed with global warming, so the first frost frequently occurred before maturity. When the average daily temperature of five successive days lower than $16^{\circ} \mathrm{C}$ occurred before DVS $=1.9$ (development stage), there was a severe impact on WSO. The years with above situation were consistent with those actually occurred in historical record. The partial early first frost occurred mainly in the period of $1.8<\mathrm{DVS} \leq 1.9$ in the Northeast. The numbers of sites where WSO reduced owing to the first frost were more than $11 \%$ in the Northeast for $44 \%$ of years. The years that the first frost occurred before maize maturity resulted in halt of WSO accumulation were consistent with historical record. It is concluded that low temperature in the grain filling period and the
\end{abstract}

本研究由国家公益性行业(气象)科研专项(GYHY200906022), 中国气象科学研究院基本科研业务费专项(2009Y005)和国家科技支撑计 划项目(2006BAD04B02)资助。

*通讯作者(Corresponding author): 马玉平, E-mail: mayp@cams.cma.gov.cn

Received(收稿日期): 2011-01-26; Accepted(接受日期): 2011-05-26; Published online(网络出版日期): 2011-06-28.

URL: http://www.cnki.net/kcms/detail/11.1809.S.20110628.1011.020.html 
earlier first frost are the key factors of maize chilling disaster in the Northeast, which could be used as the indicators in earlier warning and prediction of chilling disaster based on the crop growth model output. Meanwhile, the crop growth model can be a powerful tool to analyze agro-meteorological disasters factors.

Keywords: Maize chilling disaster; Low temperature in grain filling period; First frost; Crop growth model

20 世纪 60、70 年代, 我国东北玉米生产常因遭 受严重低温冷害而产量不稳 ${ }^{[1-5]}$ 。近些年来, 尽管全 球气候变暖, 东北地区的气温也明显上升, 但由于 普遍存在玉米相对晚熟品种越区(北扩)种植现象, 玉米低温冷害的发生频率和强度并不因气候变暖而 减少 ${ }^{[6]}$, 区域性和阶段性的玉米低温冷害仍时有发 生。从发生时段来看, 东北玉米冷害的致灾因子主 要有营养生长期低温、生殖生长期低温和秋季初霜 冻。营养生长期低温可延迟玉米抽雄期, 生殖生长 期低温会滞缓或终止玉米灌浆, 两者均可造成玉米 在初霜冻前不能正常成熟和籽粒含水量偏高。不同 年份玉米低温冷害的成因并不相同, 研究冷害的致 灾因子及其影响程度可为防灾减灾提供科学依据。

固然可以开展田间试验或人工气候箱控制试验 研究玉米的致灾因子, 但前者裸露于大气中难以实 现低温控制, 后者常因试验条件受限且难以消除与 大田实际的差异, 加之两者都有成本高、周期长的 问题, 因此采用作物模拟方法开展玉米低温冷害研 究成为一种新的途径。作物生长模型是一种面向生 长过程的机理性模型, 它可以模拟辐射、温度和水 分等环境因子对作物物候发育、光合作用、呼吸作 用、干物质积累以及产量形成过程的影响, 并在一 定程度上反映低温与作物生长发育之间的定量关 系。近些年来, 随着作物生长模型研究的深入发展, 已有学者将其引入作物低温冷害研究 ${ }^{[7-9]}$, 如通过模 拟玉米发育进程分析营养生长期低温对玉米抽雄期 的影响 ${ }^{[7-8]}$ 。但利用作物生长模型进行致灾因子研究, 特别是针对生殖生长期低温和初霜冻对玉米生长发 育及产量形成影响的研究还鲜见报道。

本研究利用作物生长模型分析东北地区玉米灌 浆期低温和初霜冻等冷害致灾因子的发生规律及其 对玉米生长发育的影响, 探讨这两种致灾因子与实 际冷害之间的数量关系, 以期为进一步探讨玉米低 温冷害监测预测方法, 开展冷害监测预测提供依据。

\section{1 数据与模型}

选取吉林白城和榆树 2 个农业气象试验站 12 年 (1994-2005)的玉米分器官生物量观测数据和东北 三省(黑龙江、吉林和辽宁) 44 个农业气象观测站 25 年(1980-2005)的玉米发育期数据, 共 923 份样本; 以及对应 44 个气象观测站 1961-2006 年逐日最高、 最低、平均温度、降水量、日照时数、水汽压和风 速等。

另外, 依据文献[2]和 [5], 并对国家气候中心 1982-2006 年气候影响评价公报分析总结, 归纳确 认了东北地区实际出现的一般性和严重玉米低温冷 害的年份(表 1)。可以看出, 20 世纪 60 80 年代, 东 北地区大约每两年即出现 1 次玉米低温冷害, 而 90 年代共出现 2 次冷害, 之后还未发生过较大范围的 玉米冷害。

采用以荷兰瓦赫宁根系列作物模型 WOFOST ${ }^{[10]}$ 、 SUCROS $^{[11]}$ 等的基本框架修改重建的“东北玉米生 长模型” (NEC_MaGM) $)^{[7-8]}$ 。该模型以日为时间步长, 由发育、生长(光合、呼吸、同化物分配及叶面积增 长)、根伸长、蒸散、土壤水分平衡等子模式组成。 其中, 发育子模式是根据玉米发育速度与最高、最 低温度的定量关系采用热量单位(CHU)来模拟发育 进程(式 1 式 3), 并以发育指数(DVS)表示。出苗期、 抽雄期和成熟期的 DVS 定义为 $0 、 1$ 和 2, 其他如吐 丝期和乳熟期的 DVS 约为 1.1 和 1.5。生长子模式 依据光合作用、维持呼吸作用以及叶面积增长等对 温度的响应模拟温度变化对生物量积累的影响。

$$
\begin{gathered}
\text { CHU }=\frac{T_{\min }^{\prime}+T_{\max }^{\prime}}{2} \\
T_{\min }^{\prime \prime}=T_{\min }-4.4 \\
T_{\max }^{\prime \prime}=a\left(T_{\max }-10\right)-b\left(T_{\max }-10\right)^{2}
\end{gathered}
$$

式中, $T_{\max } 、 T_{\min }$ 分别为日最高、最低气温 $\left({ }^{\circ} \mathrm{C}\right)$ 。 $a 、 b$ 为参数, 分别取 2 和 $0.05\left({ }^{\circ} \mathrm{C}^{-1}\right)$ 。

表 1 东北地区实际出现玉米低温冷害的年份

Table 1 Appeared years of maize chilling injury in northeast China

\begin{tabular}{ll}
\hline \multicolumn{1}{c}{ 冷害等级 Chilling class } & \multicolumn{1}{c}{ 年份 Year } \\
\hline 严重冷害 Severe chilling & $1966,1969,1971,1972,1976,1979,1980,1987,1992,1995$ \\
一般冷害 Moderate severe chilling & $1964,1965,1968,1974,1981,1986,1989$ \\
\hline
\end{tabular}




\section{2 研究结果}

\subsection{NEC_MaGM 的模拟检验}

以往研究将东北玉米发育参数(CHU)划分为 5 个区域 ${ }^{[8]}$ 。本研究利用覆盖黑龙江、吉林和辽宁三 省更多的玉米发育期共 923 份数据, 重新对 NEC $\mathrm{MaGM}$ 中的发育参数进行区域划分。首先根据各站 出苗至成熟期间 $\mathrm{CHU}$ 的多年平均累积值将东北玉 米种植区划分为 11 个区域，并参考有关与玉米熟性 密切相关的玉米叶片数的等值线走向和地形情况 ${ }^{[2]}$ 作进一步修正(图 1)。然后根据实测数据确定各分区 发育参数值。

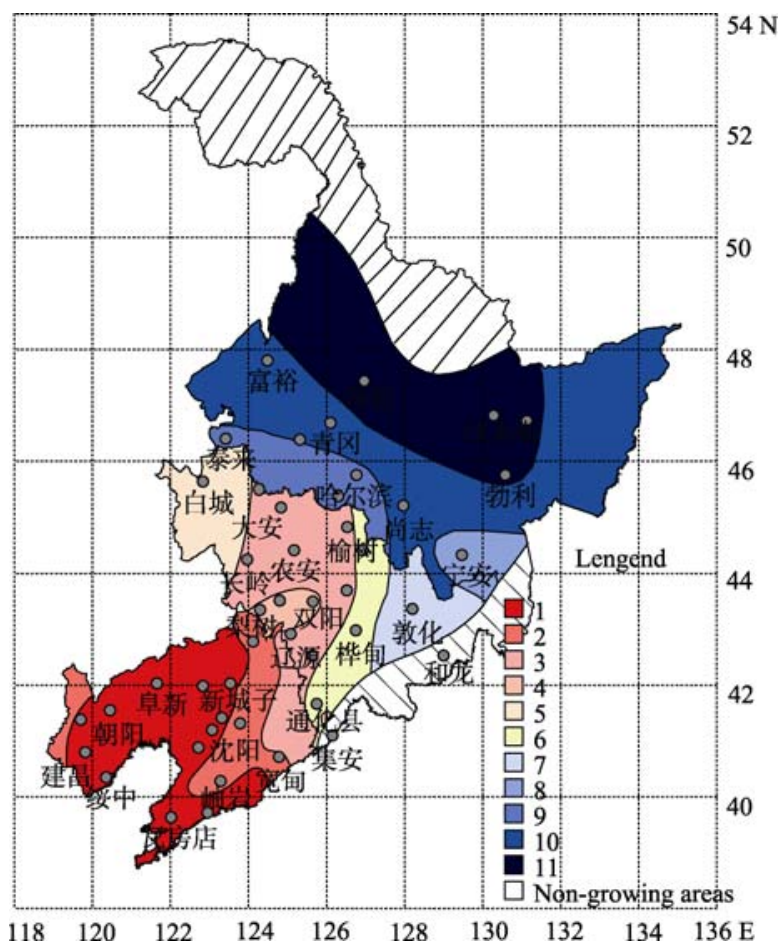

图 1 东北玉米模型 $(\mathrm{NEC} M a G M)$ 的发育参数分布图

Fig. 1 Distribution of the development parameter in North East China Maize Growth Model (NEC_MaGM)

利用黑、吉、辽三省实测数据对 NEC_MaGM 模拟发育期的效果进行检验, 并按式(4) (6)分别计 算了偏差 (Bias)、绝对偏差 (ABS) 和均方根差 (RMSE)。结果表明，抽雄期、成熟期的模拟值与实 测值的相关系数分别为 0.69 和 0.57 , 均在 0.01 统计 水平上显著(图 2); 模拟抽雄期的偏差为 $-0.3 \mathrm{~d}$, 成 熟期的为 $-1.8 \mathrm{~d}$, 表明模拟发育期总体上稍偏早; 模 拟抽雄期的绝对偏差为 $3.6 \mathrm{~d}$, 均方根差为 $0.16 \mathrm{~d}$, 模拟成熟期的绝对偏差为 $5.9 \mathrm{~d}$, 均方根差为 $0.26 \mathrm{~d}$, 在生产上均能被接受。抽雄期的模拟效果优于成熟
期，这是因为东北玉米发育后期温度较抽雄期相对 偏低，因而累积相同的 CHU 需要更多天数。

$$
\begin{aligned}
\text { Bias } & =\frac{1}{n} \sum_{i=1}^{n}\left(P_{i}-O_{i}\right) \\
A B S & =\frac{1}{n} \sum_{i=1}^{n}\left|P_{i}-O_{i}\right| \\
R M S E & =\sqrt{\sum_{i=1}^{n}\left|\left(P_{i}-O_{i}\right)^{2}\right|} / n
\end{aligned}
$$

式中, $P_{i}$ 为模拟值, $O_{i}$ 为实测值。

在上述玉米参数分区的基础上，利用白城和榆 树农业气象试验站各 12 年(1994-2005)的生物量观 测数据对 NEC_MaGM 的生长子模块进行可靠性检 验(图 3), 总体误差见表 2。从全生育期不同时段的 模拟结果看，地上总干重(TAGP)的模拟效果较好, 两站的均方根差都在 $756.8 \mathrm{~kg} \mathrm{hm}^{-2}$ 以内; 而榆树咜 存器官干重 (WSO) 的模拟值有所偏高, 偏差为 $2880.1 \mathrm{~kg} \mathrm{hm}^{-2}$, 这可能与生长子模块对水分胁迫 的模拟效果稍差有关。

\section{2 玉米灌浆期低温致灾分析}

玉米为喜温作物，对温度的要求较高。据前人 研究，玉米籽粒灌浆到成熟阶段的适宜日平均温度 为 $22 \sim 24^{\circ} \mathrm{C}$ 。当日均温低于 $15 \sim 16^{\circ} \mathrm{C}$ 时, 玉米的生理 代谢会发生紊乱，灌浆过程基本停此 ${ }^{[10-11]}$ 。若低温 出现早，持续时间长，对玉米灌浆的影响更大。

2.2 .1 东北玉米灌浆期平均温度的时空分布 首 先利用 NEC_MaGM 模拟 1961-2006 年东北各地玉 米灌浆期的起止时间(本文取吐丝期至成熟期), 然 后根据相应历史气象数据计算该时段的平均温度。 结果表明，东北玉米灌浆期的多年平均温度在 16.7 $23.5^{\circ} \mathrm{C}$ 之间, 最小值出现在黑龙江省海伦 $\left(16.7^{\circ} \mathrm{C}\right)$ 。 黑龙江、吉林和辽宁省玉米灌浆期的多年平均温度 分别为 $18.8^{\circ} \mathrm{C} 、 20.0^{\circ} \mathrm{C}$ 和 $21.9^{\circ} \mathrm{C}$ 。但从日变化来看, 不同年份不同地点的差异很大，一些年份在玉米灌 浆期屡屡出现 $15 \sim 16^{\circ} \mathrm{C}$ 以下的急剧降温天气。如出 现严重低温冷害的 1976 年, 东北地区有 $20.5 \%$ 站点 全灌浆期的平均温度低于 $17^{\circ} \mathrm{C}$, 其中黑龙江省高达 $60 \%$ 。1 995 年东北全区玉米灌浆期平均气温低于 $17^{\circ} \mathrm{C}$ 的站点占 $25 \%$, 黑龙江省也高达 $60 \%$ 。至于部 分时段低于 $16^{\circ} \mathrm{C}$ 以下的站次则更多。由此可见，灌浆 期低温对东北玉米生长和产量形成的影响不容忽视。

2.2.2 东北玉米灌浆期低温与冷害年份的对应关系 玉米灌浆期遭受冷害的严重程度不仅与低温出 

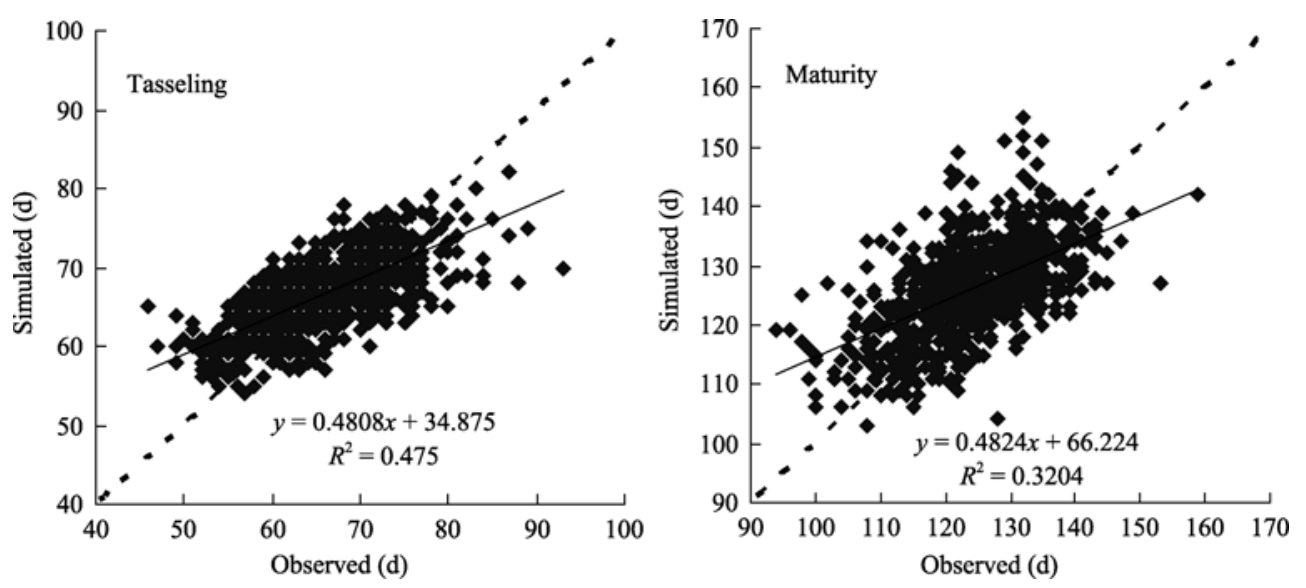

图 2 东北玉米生长模型(NEC_MaGM)模拟发育期与实测发育期(出苗后天数)的对比

Fig. 2 Comparisons between the observed and simulated development periods (in days after emergence) using NEC_MaGM 图中虚线为 $1: 1$ 线, 实线为趋势线。

Dotted line is 1:1 line; solid line is trend line.
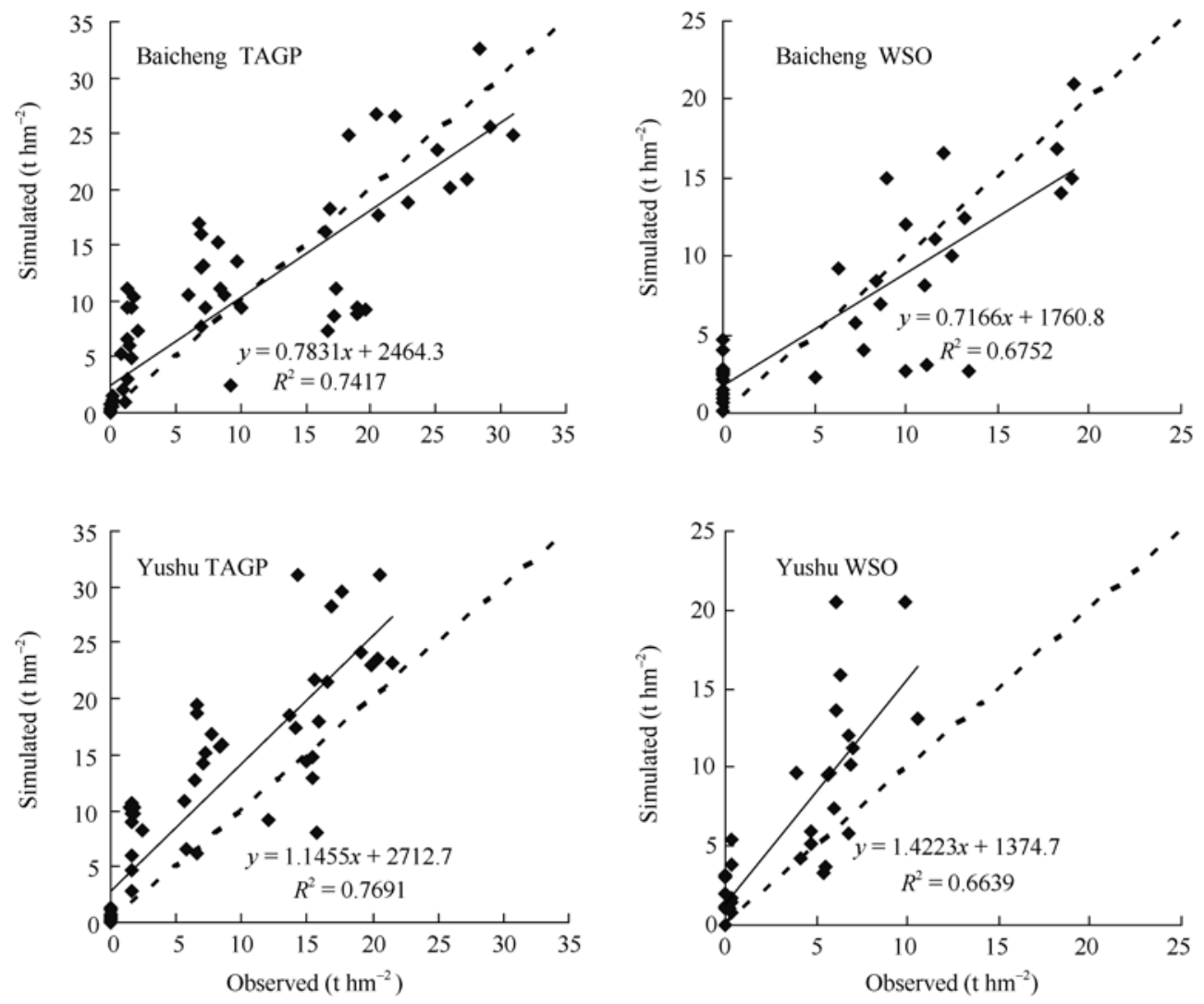

图 3 东北玉米生长模型(NEC_MaGM)模拟的生物量和实测值的对比

Fig. 3 Comparisons of observed biomass with simulated biomass by NEC_MaGM

WSO: 财存器官干重, TAGP: 地上总干重。

WSO: dry weight of storage organs; TAGP: total dry weight of maize above ground.

表 2 东北玉米生长模型(NEC_MaGM)的生物量模拟误差

Table 2 Errors of maize biomass simulated by NEC_MaGM $\left(\mathrm{kg} \mathrm{hm}^{-2}\right)$

\begin{tabular}{lccccccc}
\hline \multirow{2}{*}{$\begin{array}{c}\text { 站点 } \\
\text { Site }\end{array}$} & \multicolumn{2}{c}{ 咜存器官干重 Dry weight of storage organs } & & \multicolumn{3}{c}{ 地上总干重 } & Total dry weight of maize above ground \\
\cline { 2 - 3 } & 偏差 Bias & 绝对偏差 ABS & 均方根差 RMSE & & 偏差 Bias & 绝对偏差 ABS & 均方根差 RMSE \\
\hline 吉林白城 Baicheng, Jilin & -223.8 & 2834 & 651.6 & & 737.1 & 3517 & 605.5 \\
吉林榆树 Yushu, Jilin & 2880.1 & 3184 & 839.5 & & 3640 & 4104 & 756.8 \\
\hline
\end{tabular}


现的早晚和强度有关, 也与持续时长有关。本文利 用 NEC_MaGM 和历史实际冷害出现的年份数据, 主要探讨了灌浆期低温 $\left(<16^{\circ} \mathrm{C}\right)$ 出现早晚和持续天数 与冷害发生年份之间的关系。

首先计算了自玉米吐丝期开始东北地区各站历 年第一次出现连续 $n$ 天 $(n=1, \ldots, 7)$ 日均温 $<16^{\circ} \mathrm{C}$ 的 初日及所处的发育阶段, 并统计了持续不同天数的 低温在 DVS $\leq 1.9$ 期间出现的频次。与历史实际冷 害年份对比发现, 如果选用连续 $4 \mathrm{~d}$ (或以下)出现日 均温 $<16^{\circ} \mathrm{C}$ 的年份作为低温年, 则一些非低温冷害 年份也被包括进来; 若选用第一次连续 $5 \mathrm{~d}$ 出现日 均温 $<16^{\circ} \mathrm{C}$ 且 $\mathrm{DVS} \leq 1.9$ 、站点数目较多 (63\%以上) 的年份作为低温年，则包括了 1966、1972、1976、 1981、1995 年 5 个典型冷害年; 而选用低温持续天 数 $\geq 6 \mathrm{~d}$, 则部分历史冷害年份如 1972、1995 年就会 被遗漏。由此可见, 选用 DVS $\leq 1.9$ 且第一次出现连 续 $5 \mathrm{~d}$ 日均温 $<16^{\circ} \mathrm{C}$ 的指标与历史记载的大范围冷害 年份有较好的对应关系。

进一步分析了灌浆期低温造成玉米 WSO 损失 与出现冷害年份之间的关系。利用 NEC_MaGM 模 拟了灌浆期连续出现 $n$ 天 $(n=1, \ldots, 7)$ 日均温 $<16^{\circ} \mathrm{C}$ 时的 WSO 及其与模拟成熟期(仅根据发育参数)时 WSO 的差值百分比。从 44 站 WSO 平均差值百分比 看(图 4), 灌浆期出现持续 $3 \mathrm{~d}$ 以下低温的概率大、 时间早, 模拟若在此时终止, 则对 WSO 积累影响较 大, 大多数年份 WSO 差值百分比在 $5 \%$ 以上。而出 现连续 4 7 d 低温时累积 WSO 降低 1\% 3\%, 损失较 大年份为 1972、1976、1981 和 1995 年, 与历史严 重冷害年记载一致。
总体来看, 根据 NEC_MaGM 的数值模拟结果 并结合历史冷害数据可以认为, 当 DVS $\leq 1.9$ 时若 出现连续 $5 \mathrm{~d}$ 日平均温度 $<16^{\circ} \mathrm{C}$ 的低温使 WSO 累积 产生损失, 将导致东北玉米冷害大面积发生, 是重 要的冷害致灾因子。

\section{3 秋季初霜冻致灾分析}

秋季初霜冻过早来临, 轻者可造成尚在生长中 的玉米叶片失水萎蒸, 重者使植株枯死而不能正常 成熟, 最终导致减产。对东北各地初霜冻日(本文以 日最低气温 $\leq 2^{\circ} \mathrm{C}$ 为霜冻)的多年平均温度分析表明, 黑龙江、吉林和辽宁分别在 $7.4 \sim 8.0^{\circ} \mathrm{C} 、 7.8 \sim 9.2^{\circ} \mathrm{C}$ 和 8.4 9. $0{ }^{\circ} \mathrm{C}$ 之间, 均低于玉米生长的下限温度 $10^{\circ} \mathrm{C}^{[10]}$; 从初霜冻日出现时间的多年平均来看, 东北各地由 东北至西南方向依次出现; 从年际变化来看, 多数 地区初霜冻日均有推迟的趋势, 且吉林大多数地区 年际波动较大, 黑龙江大部分地区自 20 世纪 90 年 代以来年际间波动加大。本节将利用 NEC_MaGM 探讨过早出现的初霜冻对玉米生长发育的影响及与 冷害发生年份之间的对应关系。

\subsection{1 初霜冻出现时间与玉米冷害年份的对应关系}

利用 NEC_MaGM 模拟了东北地区 44 个台站 46 年的玉米成熟期, 并以之与各地初霜冻日作时间早 晚的比较。结果表明, 初霜冻日出现在玉米成熟期 之前的年份在辽宁省仅占 $28 \%$, 且不普遍, 而在黑 龙江、吉林两省初霜冻日出现在成熟期前的年份多 达 76\%和 $74 \%$, 并带有普遍性。可见对后两个省来 说, 存在较大的初霜冻危害。

进一步分析发现, 黑龙江初霜冻日早于成熟期 的天数最多, 大多为 $4 \sim 12 \mathrm{~d}$ 。全省有 5 年 $80 \%$ 以上

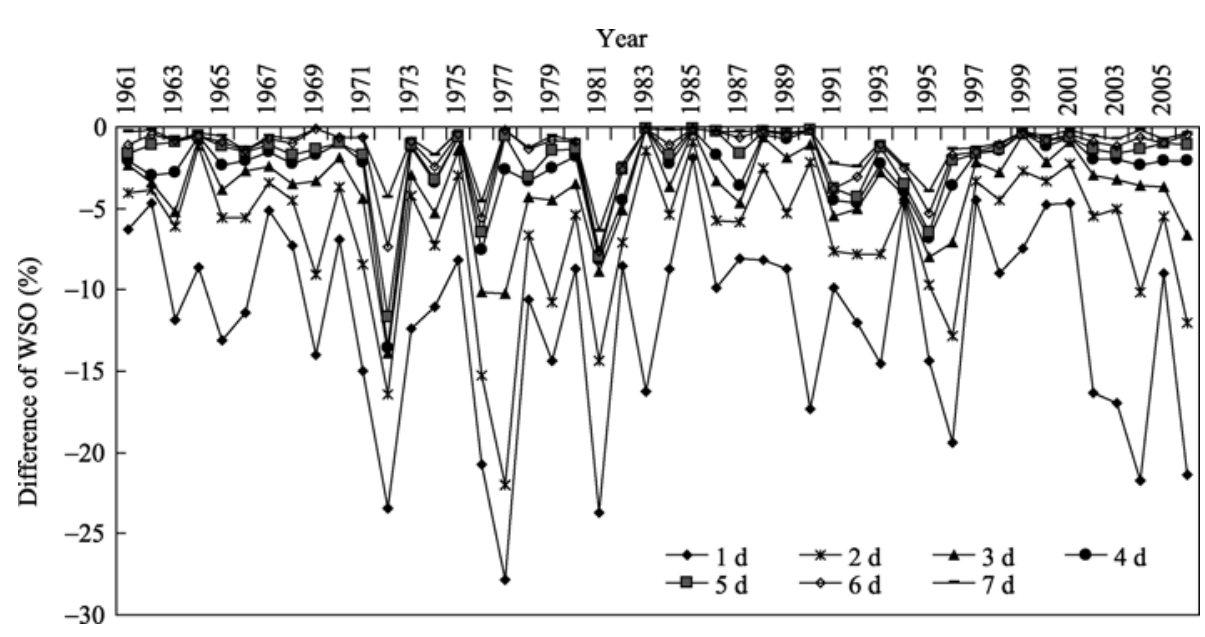

图 4 玉米灌浆期持续低温 $\left(<16^{\circ} \mathrm{C}\right)$ 对 WSO 积累的影响(图例为持续天数)

Fig. 4 Influence of continuous low temperature $\left(<16^{\circ} \mathrm{C}\right)$ on accumulation of WSO during filling stage (legend shows continuous days) 
的台站初霜冻日都出现在成熟期前, 均发生在 20 世 纪 90 年代。另有 5 年 $60 \%$ \% $0 \%$ 的台站初霜冻日出 现在模拟成熟期前, 主要发生在 60 和 70 年代。吉 林初霜冻日早于成熟期的天数比黑龙江略少, 约为 $2 \sim 10 \mathrm{~d}$ 。其中, 1992 年 $80 \%$ 以上的台站都出现在成熟 期前。1 1967 和 1995 年有 $60 \%$ 80\%的台站初霜冻日 出现在成熟期前。可以看出, 尽管东北地区 20 世纪 90 年代较 $60 、 70$ 年代气候变暖, 但初霜冻发生在玉 米成熟前的次数反而有增无减, 初霜冻对玉米的影 响不容忽视。

概括地说, 东北各地初霜冻日发生在 NEC_ $\mathrm{MaGM}$ 模拟成熟期之前、且提前天数平均在 $5 \mathrm{~d}$ 以 上的年份有 1964、1966、1967、1969、1972、1976、 $1981 、 1992 、 1993 、 1995 、 1996 、 1997 、 1999$ 年等, 其中 $62 \%$ 的年份与实际冷害大面积发生的年份一致 (图 5)。表明霜冻出现较早是东北玉米发生严重冷害 的原因之一。

\subsection{2 偏早初霜冻出现频次与玉米冷害年份的对应} 关系初霜冻日出现在不同发育阶段对玉米 WSO 积累有不同影响。统计了东北地区 44 个台站 历年在不同发育阶段发生初霜冻的频次。结果表明, 初霜冻在发育指数 DVS $\leq 1.8$ 的各阶段共出现 28 站 次, 在 $1.8<\mathrm{DVS} \leq 1.9$ 各阶段共出现 94 站次。

从省际间的比较可以看出, 黑龙江初霜冻日出 现在 DVS $\leq 1.8$ 各阶段的有 15 站次, 吉林 11 站次, 辽宁 2 站次。初霜冻日出现在 $1.8<\mathrm{DVS} \leq 1.9$ 各阶段 的, 黑吉辽依次有 $48 、 40$ 和 6 站次。可见, 历史上 出现于 $1.8<\mathrm{DVS} \leq 1.9$ 阶段的偏早初霜冻最多, 且 主要发生在黑龙江和吉林两省。

在历史典型冷害年份(1966、1969、1972、1976、 1979、1980、1985、1995 年)中, 黑龙江、吉林 2 省 有 5 年(1966、1969、1972、1976、1995)初霜冻出现
较早(DVS $\leq 1.9)$, 站点数占 $10 \%$ 46\%。

2.3.3 初霜冻对 WSO 的影响及其与冷害年份的对 应关系利用 NEC MaGM 分析了出现在模拟成 熟期之前的霜冻对玉米 WSO 的影响。结果表明, 东 北地区有 $44 \%$ 的年份因霜冻而导致 WSO 减少的站 点在 $11 \%$ 以上。一些典型冷害年份, 如 1967 年东北 地区有 22 站因初霜冻发生较早而 WSO 减少 $1 \%$ \% , 其中吉林减少的站点占全省总站数的 $61 \%$ ，黑龙江 占全省的 $60 \%$ 。1995 年东北地区有 18 站因初霜冻 导致 WSO 减少, 有 13 站减少 6\% 9\%。1972 年因初 霜冻导致 WSO 减少 8 站, 且减少幅度均大于 $5 \%$, 尽 管范围小，但损失大。

从东北地区多站平均来看, 出现在模拟成熟期 之前的霜冻造成累积 WSO 数量减少 $1.5 \%$ 以上的年 份有 1961、1966、1967、1972、1978、1981、1995 和 2006 年。

总体来看, 利用 NEC_MaGM 分析玉米成熟前 出现初霜冻对玉米生长发育影响比较严重的年份与 历史上出现冷害的一些典型年份(如 1966、1969、 1972、1976、1985、1995 年)比较一致。因此, 初霜 冻的发生时间及导致 WSO 的损失程度是冷害的重 要致灾因子之一。

\section{3 讨论}

玉米气象致灾因子研究一般可通过田间试验或 人工气候箱试验开展 ${ }^{[12-15]}$ 。利用作物生长模型模拟 的方法开展相关研究可克服以上试验手段难以控 制、成本高且周期较长的不足。本文利用作物生长 模型开展东北玉米生殖生长期的冷害致灾因子研究, 不仅拓宽了模型的应用渠道, 也为相关研究提供了 新的工具。但是致灾研究对作物生长模型的模拟精 度有较高要求。本研究中所用 NEC_MaGM 模型经

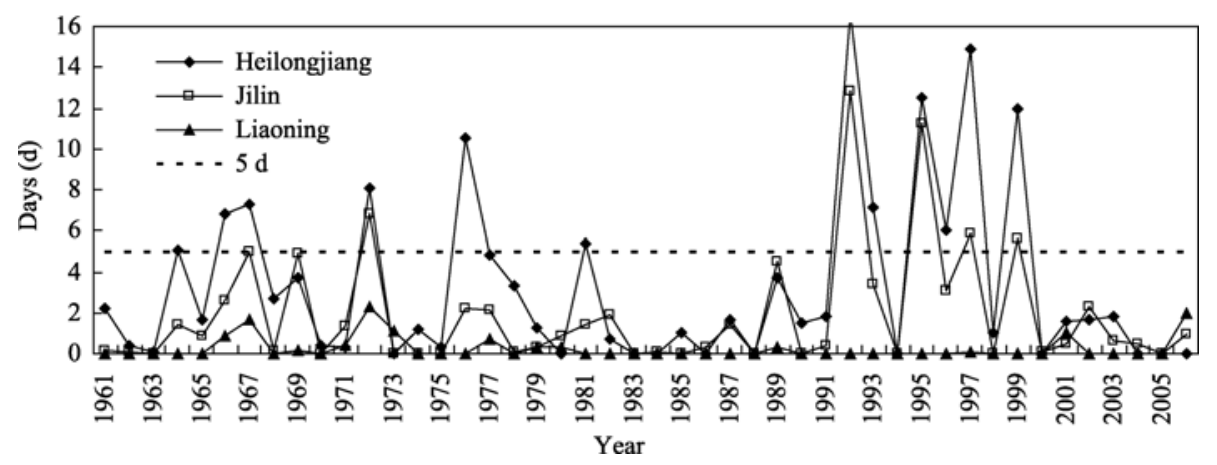

图 5 东北地区初霜冻日距玉米模拟成熟期的天数

Fig. 5 Days between the first frost date observed and the maturity simulated in northeast China 
参数调整后的绝对误差, 模拟抽雄期为 $3.6 \mathrm{~d}$, 成熟 期的为 $5.9 \mathrm{~d}$, 两个典型站点穗重模拟的均方根误差 在 $839.5 \mathrm{~kg} \mathrm{hm}^{-2}$ 以内。通过计算相对影响分析冷害 致灾因子尽可能地减少了系统误差。另外，抽雄期 的模拟效果好于成熟期, 为下一步选择抽雄期为冷 害指标提供良好条件。今后的研究中还应进一步改 进模型对成熟期和穗重的模拟效果，并加强水分胁 迫影响的模拟。

霜冻是指作物生长期间土壤表面或作物冠层附 近的最低温度降到 $0^{\circ} \mathrm{C}$ 以下, 导致作物体内结冰、生 理活性受损或冰晶挤压发生机械损伤的现象。限于 地面或冠层温度数据较难获得, 许多研究用日最低 温度代替。一般情况下, 发生霜冻时, 日最低温度比 地表温度高 $2 \sim 5^{\circ} \mathrm{C}^{[10]}$ 。有的研究用日最低气温 $\leq 0^{\circ} \mathrm{C}$ 代替 ${ }^{[16-17]}$, 较多研究一般用日最低气温 $\leq 2^{\circ} \mathrm{C}$ 代替 ${ }^{[3,18]}$, 对黄淮麦区的研究发现霜冻日的最低气温在 $-6.1 \sim 1.3^{\circ} \mathrm{C}$ 之间 ${ }^{[19]}$ 。可见, 地表或冠层温度与日最低 气温常不等价。这种对应关系还与霜冻类型有关, 对于平流型霜冻, 两者的差距较小; 但对于辐射型 霜冻, 两者相差可能大于 $2^{\circ} \mathrm{C}$, 甚至达 $5^{\circ} \mathrm{C}^{[10]}$ 。因此, 本文利用日最低气温建立霜冻与冷害的关系尚不够 细致, 未来还可利用地面最低温度进一步研究霜冻 与冷害的关系。

由于作物生育期内气象条件影响的复杂多样性 和不同生长阶段的前后连续性、互补性, 在不同年 份玉米抽雄至成熟期间的光、热、水特别是温度条 件的好或差有可能加快或延迟玉米的发育进程, 从 而起到减轻或加剧前期不利温度条件的影响程度。 因此, 导致玉米发生低温冷害的因子除了营养生长 阶段温度偏低外, 还包括灌浆期温度偏低、秋季初 霜冻过早来临等造成发育进程或干物质积累停止等 后期不利热量条件。这些因素都可以作为利用作物 生长模型进行玉米冷害监测预测的重要指标。

\section{4 结论}

东北地区玉米灌浆期平均温度一般在 16.7 $23.5^{\circ} \mathrm{C}$ 之间, 但一些年份仍屡屡出现 $15 \sim 16^{\circ} \mathrm{C}$ 以下 (灌浆受阻温度)的急剧降温天气, 对东北玉米生长 有较大影响。东北地区初霜冻日的平均温度在 7.4 $9.2^{\circ} \mathrm{C}$ 之间, 已经低于玉米生长下限。初霜冻日由东 北向西南方向依次出现, 各地初霜冻日随年份增加 均有推迟的趋势，但年际间波动也在加大。尽管东 北气候逐渐变暖, 但由于新的玉米品种成熟期不断
推迟，初霜冻发生在玉米成熟前的频率在 20 世 纪 90 年代相比前 20 30 年反而有所增加。

NEC_MaGM 的模拟试验表明，灌浆期连续 $5 \mathrm{~d}$ 日平均温度低于 $16^{\circ} \mathrm{C}$, 同时出现在 $\mathrm{DVS} \leq 1.9$ 时对 玉米储存器官干重积累造成较大影响的年份与实际 冷害发生年份比较相符, 是东北玉米发生大面积冷 害的致灾因子之一; 玉米成熟前的初霜冻对玉米 WSO 积累影响比较严重的年份与历史上出现冷害 的一些典型年份比较一致, 初霜冻出现时间及其造 成 WSO 的损失程度也是冷害的致灾因子之一。由此 也可看出，作物生长模型可以成为研究农业气象灾 害致灾因子的有力工具。

\section{References}

[1] Sun Y-T(孙玉亭), Wang S-Y(王书裕), Yang Y-Q(杨永岐). Studies on cool summer and crop yield in northeast China. Acta Meteorol Sin (气象学报), 1983, 41(3): 313-321 (in Chinese with English abstract)

[2] Feng P-Z(冯佩芝), Li C-J(李翠金), Li X-Q(李小泉). Analysis of China’s Major Meteorological Disasters during 1951-1980 (中国 主要气象灾害分析 1951-1980). Beijing: China Meteorological Press, 1985. pp 1-271 (in Chinese)

[3] Zhang Y-C(张养才), He W-X(何维勋), Li S-K(李世奎). Introduction to Agricultural Meteorological Disasters in China (中国 农业气象灾害概论). Beijing: China Meteorological Press, 1991. pp 24-259 (in Chinese)

[4] Wang S-Y(王书裕). Studies on Crop Chilling Disaster (农作物冷 害的研究). Beijing: China Meteorological Press, 1995. pp 1-246 (in Chinese)

[5] Mao F(毛飞), Gao S-H(高素华), Wang C-Y(王春乙). Studies on energy resources and chilling disaster in northeast China. Acta Meteorol Sin (气象学报), 2000, 58(suppl): 871-880 (in Chinese with English abstract)

[6] Fang X-Q(方修琦), Wang Y(王媛), Zhu X-X(朱哓禧). Change of cool summer hazard under an adaptation behavior to the climate warming in Heilongjiang province, northeast China. Geographical Res (地理研究), 2005, 24(5): 664-672 (in Chinese with English abstract)

[7] Liu B-C(刘布春), Wang S-L(王石立), Zhuang L-W(庄立伟), Lu Z-G(卢志光), Shi X-L(史学丽), Song Y-J(宋永佳). Study of low temperature damage prediction applications in EN, China based on a scaling-up maize dynamic model. J Appl Meteorol (应用气象 学报), 2003, 14 (5): 616-625 (in Chinese with English abstract)

[8] Wang S-L(王石立), Ma Y-P(马玉平), Zhuang L-W(庄立伟). Improvement study on prediction and assessment model for chilling damage of maize in northeast China. J Nat Disasters (自 然灾害学报), 2008, 17(4): 12-18 (in Chinese with English abstract)

[9] Ma S-Q(马树庆)，Liu Y-Y(刘玉英)，Wang Q(王琪). Dynamic 
prediction and evaluation method of maize chilling damage. Chin $J$ Appl Ecol (应用生态学报), 2006, 17(10): 1905-1910 (in Chinese with English abstract)

[10] Beijing Agricultural University Agrometeorological Speciality (北京农业大学农业气象专业). Agrometeorology (农业气象学). Beijing: Science Press, 1984. pp 192-319 (in Chinese)

[11] Setter T L, Flannigan B A. Sugar and starch redistribution in maize in response to shade and ear temperature treatment. Crop Sci, 1986, 26: 575-579

[12] Wang Q(王琪), Ma S-Q(马树庆), Guo J-P(郭建平), Zhang T-L(张铁林), Yu H(于海), Xu L-P(徐丽萍). Effects of air temperature on maize growth and its yield. Chin Ecol (生态学杂志), 2009, 28(2): 255-260 (in Chinese with English abstract)

[13] Guo J-P(郭建平), Wang C-Y(王春乙), Ma S-Q(马树庆), Wang $\mathrm{Q}$ (王琪), Shen Z-S(沈钟声). Experimental study on defending corn from cold injury and spring drought by growing and transplanting seedlings. Nat Disasters (自然灾害学报), 2003, 12(1): 116-120 (in Chinese with English abstract)

[14] Fang W-J(房稳静), Wu J-H(武建华), Chen S(陈松), Zhang J(张 静), Li C(李晨), Liu R(刘端). Experiment for the impact of flood in different development stages on summer maize growth and yields. Chin Agrometeorol (中国农业气象), 2009, 30(4): 616618 (in Chinese with English abstract)
[15] Wang R(王瑞), Ma F-M(马凤鸣), Li C-F(李彩凤), Chen S-Y(陈 胜勇), Hou J(侯静). Effect of low temperature stress on proline, malondialdehyde contents and electric conductivity of maize seedling. Northeast Agric Univ (东北农业大学学报), 2008, 39(5): 20-23 (in Chinese with English abstract)

[16] Liu L(刘丽), Wang X-H(王晓红), Huang X-H(黄晓慧), Xu F-K(徐方奎), Zhao H-Y(赵慧颖), Xie X-L(谢晓丽). Features analysis of frost in the Southeastern Region of the Daxing'anling Mountains in Inner Mongolia. Anhui Agric Sci (安徽农业科学), 2010, 38(26): 14492-14493 (in Chinese with English abstract)

[17] Qian J-X(钱锦霞), Wu J(武捷), Ban S-L(班胜林). Analysis of the feature of the frost events occurrence in Taiyuan from 1951 to 2008. Chin Agric Sci Bull (中国农学通报), 2009, 25(10): 287-289 (in Chinese with English abstract)

[18] China Meteorological Administration(中国气象局). Atlas of China Disastrous Weather and Climate (中国灾害性天气气候图 集). Beijing: China Meteorological Press, 2007. pp I-IV (in Chinese)

[19] Zhong X-L(钟秀丽), Wang D-L(王道龙), Zhao P(赵鹏), Yan X-Y(间旭宇), Su C-H(苏常红). Occurrence of frost temperature in Huanghuai wheat production zone after winter wheat elongation. Chin Eco-Agric (中国生态农业学报). 2007, 15(5): 17-20 (in Chinese with English abstract)

\section{欢迎订阅 2012 年《园艺学报》}

《园艺学报》是中国园艺学会和中国农业科学院蔬菜花卉研究所主办的学术期刊, 创刊于 1962 年, 刊 载有关果树、蔬菜、观赏植物、茶及药用植物等方面的学术论文、研究报告、专题文献综述、问题与讨论、 新技术新品种以及园艺研究动态与信息等, 适合园艺科研人员、大专院校师生及农业技术推广部门专业技术 人员阅读参考。

《园艺学报》是全国中文核心期刊, 被英国国际农业与生物中心数据库 $(\mathrm{CABI}) 、$ 美国化学文摘 $(\mathrm{CA}) 、$ 日本科学技术文献速报 $(\mathrm{CBST}) 、$ 、俄罗斯文摘杂志 $(\mathrm{AJ}) 、$ 、国科学引文数据库 $(\mathrm{CSCD})$ 等多家重要数据库收录。 2005 年荣获第三届国家期刊奖, 2008 年获中国科技信息所“中国精品科技期刊” 称号及武汉大学中国科学 评价研究中心“中国权威学术期刊” 称号, 2009 年获中国期刊协会和中国出版科学研究所“新中国 60 年有 影响力的期刊” 称号。根据“中国学术期刊影响因子年报(2010版)”的统计, 《园艺学报》期刊综合总被引 频次 4 699, 复合总被引频次 12 283, 期刊综合影响因子 1.069, 复合影响因子 1.910。

《园艺学报》为月刊, 每月 25 日出版。2012 年每期定价 40.00 元，全年 480.00 元。国内外公开发行，全 国各地邮局办理订阅，国内邮发代号 82-471，国外发行由中国国际图书贸易总公司承办, 代号 M448。漏订 者可直接寄款至本编辑部订购。

编辑部地址: 北京市海淀区中关村南大街 12 号 中国农业科学院蔬菜花卉研究所《园艺学报》编辑部 邮编: 100081; 电话: (010)82109523; E-mail: yuanyixuebao@126.com; 网址: http://www.ahs.ac.cn 